Synthesis and Biological Activity of $N$-2,3-dihydroxylpropyl- $N$-4-chlorobutyl Nucleoside Phosphoramidate Prodrugs

Weidong Wu and Richard F. Borch

\title{
Mean $\mathrm{GI}_{50}$ Values for Compounds 1a and $\mathbf{4}$ NCI 60 Cell Line Panel
}

\begin{tabular}{|c|c|c|c|}
\hline \multirow[b]{2}{*}{ Tumor Type } & \multirow[b]{2}{*}{ Cell Line } & \multicolumn{2}{|c|}{ GI50, uM } \\
\hline & & Cpd 1a & Cpd 4 \\
\hline Breast & $T-47 D$ & 4.4 & 52 \\
\hline Breast & ВТ-549 & 2.8 & 0.66 \\
\hline Breast & HS 578T & 0.51 & 0.46 \\
\hline Breast & MCF7 & 0.03 & $<0.01$ \\
\hline Breast & MDA-MB-435 & 6.7 & 1.6 \\
\hline Breast & NCI/ADR-RES & 0.09 & 0.78 \\
\hline CNS & U251 & 2.6 & 0.34 \\
\hline CNS & SNB-75 & 0.17 & 4.9 \\
\hline CNS & SNB-19 & 8.1 & 52 \\
\hline CNS & SF-539 & $<0.01$ & 0.07 \\
\hline CNS & SF-295 & 0.12 & 0.31 \\
\hline CNS & SF-268 & 0.03 & 0.76 \\
\hline Colon & COLO 205 & $<0.01$ & 53 \\
\hline Colon & HCC-2998 & $<0.01$ & 0.41 \\
\hline Colon & SW-620 & 17 & 0.08 \\
\hline Colon & HT29 & 3.6 & 1.7 \\
\hline Colon & KM12 & 13 & 5.1 \\
\hline Colon & НСТ-15 & 22 & 4.6 \\
\hline Colon & НCT-116 & 1.2 & 1.1 \\
\hline Leukemia & HL-60(TB) & 1.3 & 0.03 \\
\hline Leukemia & MOLT-4 & 0.59 & 1.6 \\
\hline Leukemia & RPMI-8226 & 5.2 & $<0.01$ \\
\hline Leukemia & $\mathrm{K}-562$ & 15 & 7.1 \\
\hline Leukemia & SR & 0.07 & 0.04 \\
\hline Leukemia & CCRF-CEM & 0.17 & $<0.01$ \\
\hline Melanoma & SK-MEL-2 & 17 & 77 \\
\hline Melanoma & SK-MEL-5 & 0.85 & 0.41 \\
\hline Melanoma & UACC-62 & 0.46 & 0.29 \\
\hline Melanoma & UACC-257 & 37 & 0.02 \\
\hline Melanoma & MALME-3M & 9.8 & 0.38 \\
\hline Melanoma & SK-MEL-28 & 24 & $>100$ \\
\hline Melanoma & M14 & 1.1 & 0.71 \\
\hline Melanoma & LOX IMVI & 0.07 & 0.03 \\
\hline
\end{tabular}




\begin{tabular}{llcc} 
& & \multicolumn{2}{c}{ Gl50, uM } \\
Tumor Type & Cell Line & $\frac{\text { Cpd 1a }}{35}$ & Cpd 4 \\
NSC Lung & EKVX & 7.4 & 54 \\
NSC Lung & HOP-92 & 0.21 & 0.23 \\
NSC Lung & HOP-62 & 0.03 & 0.41 \\
NSC Lung & NCl-H226 & 5.9 & $<0.01$ \\
NSC Lung & NCl-H322M & 6.5 & 0.52 \\
NSC Lung & NCl-H460 & $<0.01$ & 2.1 \\
NSC Lung & NCI-H522 & 3.8 & 0.12 \\
NSC Lung & NCl-H23 & 1.3 & 1.1 \\
& & & 2.8 \\
Ovarian & SK-OV-3 & 0.45 & \\
Ovarian & OVCAR-8 & 0.35 & $>100$ \\
Ovarian & OVCAR-3 & 1.2 & 0.18 \\
Ovarian & OVCAR-4 & 13 & 0.69 \\
Ovarian & IGROV1 & 2.2 & $>100$ \\
& & & 1.3 \\
Prostate & DU-145 & 1.1 & \\
Prostate & PC-3 & 5.8 & 0.18 \\
& & & 0.25 \\
Renal & UO-31 & 0.36 & 0.28 \\
Renal & A498 & 0.02 & 0.16 \\
Renal & 786-0 & 1.9 & 0.23 \\
Renal & TK-10 & 2.7 & 0.33 \\
Renal & SN12C & 0.21 & 0.13 \\
Renal & RXF 393 & 0.31 & 0.12 \\
Renal & ACHN & 0.04 & 0.03 \\
Renal & CAKI-1 & 0.08 &
\end{tabular}

